\title{
Nonequilibrium dynamics in the pump-probe spectroscopy of excitonic insulators
}

\author{
Tetsuhiro Tanabe ${ }^{1}$, Koudai Sugimoto ${ }^{2}$, and Yukinori Ohta ${ }^{1}$ \\ ${ }^{1}$ Department of Physics, Chiba University, Chiba 263-8522, Japan \\ ${ }^{2}$ Center for Frontier Science, Chiba University, Chiba 263-8522, Japan
}

(Dated: December 3, 2018)

\begin{abstract}
We study the nonequilibrium dynamics in the pump-probe spectroscopy of excitonic insulators using the spinless two-orbital model with phonon degrees of freedom in the time-dependent meanfield approximation. We introduce the pulse light as a time-dependent vector potential via the Peierls phase in the Hamiltonian. We find that, in the Bose-Einstein condensation regime where the normal state is semiconducting, the excitonic order is suppressed when the frequency of the pulse light is slightly larger than the band gap, while the order is enhanced when the frequency of the pulse is much larger than the band gap. We moreover find that the excitonic order is completely destroyed in the former situation if the intensity of the pulse is sufficiently strong. In the BCS regime where the normal state is semimetallic, we find that the excitonic order is always suppressed, irrespective of the frequency of the pulse light. The quasiparticle band structure and optical conductivity spectrum after the pumping are also calculated for the instantaneous states.
\end{abstract}

\section{INTRODUCTION}

Nonequilibrium dynamics induced by applying the intense laser pulse have recently been a new way of investigating a variety of quantum condensed phases. Recent achievement of the time resolution of a femto-second order enables one to perform experiments studying the ultrafast dynamics of materials. Examples include a success of observing light-induced superconductivity [1, 2] and a pump-probe measurement of melting of chargedensity-wave orders [3, 4].

The pump-probe measurement is also applicable to the study of excitonic condensation. In the excitonic phase, holes in the valence band and electrons in the conduction band form pairs called excitons, just like Cooper pairs of electrons in superconductivity, and they undergo quantum condensation at low temperatures [5, 6]. The realization of such condensation has been suggested in transition-metal chalcogenides $1 T-\mathrm{TiSe}_{2}$ [7, 8] and $\mathrm{Ta}_{2} \mathrm{NiSe}_{5}$ [9, 10]. Here, we should note that, since the spin-singlet excitonic state necessarily couples to the phonon degrees of freedom [11 15], it is difficult to single out the excitonic contributions at least in equilibrium state experiments. There are, however, some attempts to distinguish between the excitonic and phononic contributions using the nonequilibrium dynamics induced by laser pulse in $1 T-\mathrm{TiSe}_{2}[16-19]$. In $\mathrm{Ta}_{2} \mathrm{NiSe}_{5}$, Mor et al. found that the band gap can be controlled by the excitation density 20] and argued that its nonequilibrium phenomena come from the exciton dynamics [21]. Coherent order parameter oscillations caused by the induced phonons were also observed [22, 23].

The pump-probe spectroscopy experiments in the excitonic phases have been interpreted from the theoretical point of view. While the GW calculations showed that the excitonic order vanishes after applying the laser pulse in the BCS regime where the normal state is semimetallic [24], Murakami et al. 25] recently showed that the excitonic order can be enhanced by the laser pulse in the Bose-Einstein condensation (BEC) regime where the normal state is semiconducting. Tanaka et al. [26] also showed that the switching between the melting and enhancement of excitonic orders can occur when the order varies from the BCS regime to BEC regime.

Note that all of these calculations 24-26] assumed that the laser pulse excites the electrons in the valence-band orbital directly to the conduction-band orbital via the dipole transition. However, as was discussed in Ref. 27], the matrix elements of the dipole transition can be small in the case where the valence-band and conduction-band orbitals are well-localized and are spatially separated in distant positions, just as in $\mathrm{Ta}_{2} \mathrm{NiSe}_{5}$ 13]. The Peierls term, on the other hand, can survive even in such situations 27]. Thus, there is another way of treating the laser pulse, which is to introduce a time-dependent vector potential via the Peierls phase in a tight-binding Hamiltonian.

In this paper, we study the nonequilibrium dynamics of excitonic insulator states applying the time-dependent mean-field approximation to the spinless two-orbital model in one-dimension (1D) with phonon degrees of freedom, whereby we simulate the situation where an optical laser pulse is applied to the system as a pump light. Unlike preceding studies, we here introduce the pulse light as a time-dependent vector potential via the Peierls phase in the Hamiltonian of the external field, assuming the situations where the dipole matrix elements are small. We note that the spontaneous hybridization between the valence-band and conduction-band orbitals occurs in the symmetry-broken excitonic insulator state, so that the interband excitations by the Peierls mechanism can work in the present model. We thus investigate the time evolution of the excitonic order parameter in both the BEC and BCS regimes, paying particular attention to its dependence on the frequency and intensity of the laser light.

We will show that, in the $\mathrm{BEC}$ regime where the normal state is semiconducting, the excitonic order is suppressed when the frequency of the pulse light is slightly larger than the band gap, while the order is enhanced when the frequency of the pulse is much larger than the 
band gap. In the BCS regime where the normal state is semimetallic, we will show that the excitonic order is always suppressed, irrespective of the frequency of the pulse light. We will demonstrate that the excitonic order parameter oscillation occurs in agreement with experiment. We will also calculate the optical conductivity spectrum assuming a single-time, instantaneous response for a quasi-steady state after pumping and demonstrate the measurement is a useful way for probing the nonequilibrium dynamics of excitonic insulator states.

The rest of this paper is organized as follows. In Sec. II, we introduce the spinless two-orbital model, define the laser pulse light, and derive the equations of motions for the excitonic order parameters in the time-dependent mean-field approximation. In Sec. III, we present results for nonequilibrium dynamics induced by laser pulse in both BEC and BCS regimes. We also present results for the optical conductivity spectra in the nonequilibrium state. We summarize our results and discuss their experimental significance in Sec. IV.

\section{MODEL AND METHOD}

\section{A. Spinless two-orbital model}

As a minimum model for describing the spin-singlet excitonic insulator state coupled with phonon degrees of freedom, we consider the spinless two-orbital model (or extended Falicov-Kimball model 28 31]) defined on the 1D lattice [see Fig. T(a)], interacting with Einstein phonons of frequency $\omega_{0}$ [25]. This model may be relevnt to the electronic state of an excitonic insulator candidate $\mathrm{Ta}_{2} \mathrm{NiSe}_{5}$ with a quasi-1D crystal structure [10], although the method discussed below is applicable to higher dimensional systems as well. Our model is defined by the Hamiltonian

$$
H=H_{\mathrm{e}}+H_{\mathrm{e}, \mathrm{int}}+H_{\mathrm{ph}}+H_{\mathrm{e}-\mathrm{ph}}
$$

with

$$
\begin{aligned}
& H_{\mathrm{e}}=-\sum_{i, \alpha}\left(J_{\alpha} c_{i+1, \alpha}^{\dagger} c_{i, \alpha}+\text { H.c. }\right)+\sum_{i, \alpha} \Delta_{\alpha} c_{i, \alpha}^{\dagger} c_{i, \alpha} \\
& H_{\mathrm{e}, \text { int }}=U \sum_{i} c_{i, 0}^{\dagger} c_{i, 0} c_{i, 1}^{\dagger} c_{i, 1} \\
& H_{\mathrm{ph}}=\omega_{0} \sum_{i} b_{i}^{\dagger} b_{i} \\
& H_{\mathrm{e}-\mathrm{ph}}=g \sum_{i}\left(b_{i}^{\dagger}+b_{i}\right)\left(c_{i, 1}^{\dagger} c_{i, 0}+\text { H.c. }\right)
\end{aligned}
$$

where $c_{i, \alpha}\left(c_{i, \alpha}^{\dagger}\right)$ is the annihilation (creation) operator of an electron at site $i$ with orbital $\alpha(=0,1)$ and $b_{i}\left(b_{i}^{\dagger}\right)$ is the annihilation (creation) operator of a phonon at site $i$. $J_{\alpha}, \Delta_{\alpha}$, and $U$ are the hopping integral, on-site energy, and interorbital repulsive interaction, respectively, and $\omega_{0}$ and $g$ are the phonon frequency and electronphonon coupling constant, respectively. Throughout the
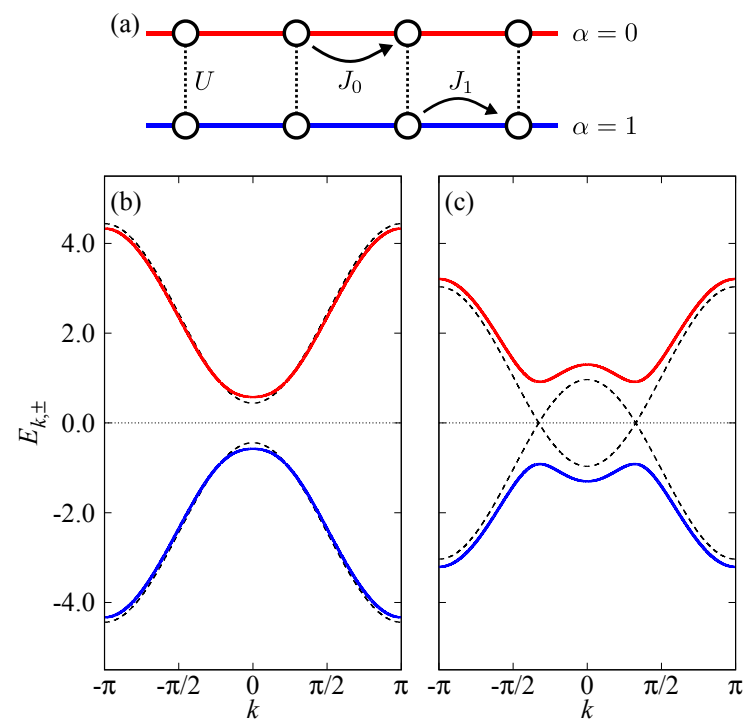

FIG. 1. (a) Schematic representation of the spinless twoband model used. In (b) and (c), we show the quasiparticle band dispersions calculated for the model (a) in the equilibrium state (solid lines), together with the noninteracting band dispersions (dotted lines). We assume $D=2.08$ in (b) and $D=1.1$ in (c).

paper, we set $J_{\alpha}=(-1)^{\alpha} J$, assuming a direct-gap semiconductor or semimetal [see Figs. 1(b) and 1(c)], so that we create a momentum $q=0$ condensate appropriate to $\mathrm{Ta}_{2} \mathrm{NiSe}_{5}$, rather than $q=\pi$ assumed in, e.g., Ref. [24]. We keep the relation $U=-\left(\Delta_{0}+\Delta_{1}\right)$, so that the condition that the number of electrons per site (containing two orbitals) is one is satisfied at the chemical potential $\mu=0$. We define the energy level difference $D=\Delta_{0}-\Delta_{1}$. We introduce the effective electron-phonon interaction $\lambda=2 g^{2} / \omega_{0}$ and assume the values $\lambda=0.1$ and $\omega_{0}=0.1$ throughout the paper. We use the values $J=1$ (unit of energy), $\Delta_{0}=-0.36, \Delta_{1}=-2.44, D=2.08$, and $U=2.8$ unless otherwise indicated. The noninteracting band dispersions used are illustrated in Figs. 1 (b) and 1(c).

\section{B. Optical laser pulse}

To treat the optical laser pulse as a pump light, we introduce the time-dependent vector potential as a Peierls phase to the Hamiltonian. We assume that the vector potential is approximately independent of the spatial position since the wavelength of the light is much longer than the lattice spacing $a$. The hopping-integral term in Eq. (22) is then replaced by [32]

$$
J_{\alpha} c_{i+1, \alpha}^{\dagger} c_{i, \alpha} \rightarrow J_{\alpha} e^{i e a A(t)} c_{i+1, \alpha}^{\dagger} c_{i, \alpha},
$$

where $e(<0)$ is the elementary charge, for which we set $e=-1$. We use the units $\hbar=c=1$ and the lattice 
constant $a=1$ throughout the paper. We consider the Gaussian-type laser pulse defined by the time-dependent vector potential as 33.

$$
A(t)=\theta(t) A_{0} e^{-\frac{\left(t-t_{\mathrm{p}}\right)^{2}}{2 \sigma_{\mathrm{p}}^{2}}} \sin \Omega t
$$

where $\Omega$ and $A_{0}$ are the frequency and intensity of the pulse light, respectively, and

$$
\theta(t)= \begin{cases}0 & (t \leq 0) \\ 1 & (t>0)\end{cases}
$$

is the step function. The time and width of the pulse are described by $t_{\mathrm{p}}$ and $\sigma_{\mathrm{p}}$, respectively. In this paper, we set $\sigma_{\mathrm{p}}=30$ and $t_{\mathrm{p}}=100$. By the Fourier transformation $c_{i, \alpha}=\frac{1}{\sqrt{N}} \sum_{k} e^{i k r_{i}} c_{k, \alpha}$, where $N$ is the number of lattice sites in the system, Eq. (2) may be rewritten as

$$
H_{\mathrm{e}}=\sum_{k, \alpha} \varepsilon_{\alpha}(k a-e a A(t)) c_{k, \alpha}^{\dagger} c_{k, \alpha}
$$

where $\varepsilon_{\alpha}(k a)=-2(-1)^{\alpha} \cos k a+\Delta_{\alpha}$.

\section{Equations of motion}

The time evolution of the system induced by the laser pulse is calculated in the time-dependent mean-field approximation [25, 34, 35]. We define the uniform excitonic order parameter as $\phi(t)=\left\langle c_{i, 0}^{\dagger}(t) c_{i, 1}(t)\right\rangle$, the uniform phonon displacement as $X(t)=\left\langle b_{i}^{\dagger}(t)+b_{i}(t)\right\rangle$, and the uniform electron density as $n_{\alpha}(t)=\left\langle c_{i, \alpha}^{\dagger}(t) c_{i, \alpha}(t)\right\rangle$. The time dependence of the operators is given by the Heisenberg representation.

For convenience, we use the pseudospin representation for the spinless electron in the two orbitals [25]. We define the pseudospin as $S_{k}^{\gamma}=\frac{1}{2} \boldsymbol{\Psi}_{k}^{\dagger} \sigma_{\gamma} \boldsymbol{\Psi}_{k}$, where $\boldsymbol{\Psi}_{k}^{\dagger}=\left(\begin{array}{ll}c_{k, 0}^{\dagger} & c_{k, 1}^{\dagger}\end{array}\right)$ is the spinor, $\sigma_{\gamma}(\gamma=x, y, z)$ is the $\gamma$ component of Pauli matrix $\boldsymbol{\sigma}$, and $\sigma_{0}$ is the identity matrix. Introducing the pseudomagnetic field as

$$
\begin{aligned}
B_{k}^{x}(t)= & 2 g X(t)-2 U \operatorname{Re} \phi(t) \\
B_{k}^{y}(t)= & -2 U \operatorname{Im} \phi(t) \\
B_{k}^{z}(t)= & {\left[\varepsilon_{0}(k a-e a A(t))-\varepsilon_{1}(k a-e a A(t))\right] } \\
& -U\left(n_{0}(t)-n_{1}(t)\right) \\
B_{k}^{0}(t)= & \sum_{\alpha}\left[\varepsilon_{\alpha}(k a-e a A(t))+U n_{\alpha}(t)\right],
\end{aligned}
$$

we may write the mean-field Hamiltonian as

$$
H^{\mathrm{MF}}(t)=H_{\mathrm{e}}^{\mathrm{MF}}(t)+H_{\mathrm{ph}}^{\mathrm{MF}}(t)
$$

with

$$
\begin{aligned}
& H_{\mathrm{e}}^{\mathrm{MF}}(t)=\sum_{k} \sum_{\gamma=0, x, y, z} B_{k}^{\gamma}(t) S_{k}^{\gamma} \\
& H_{\mathrm{ph}}^{\mathrm{MF}}(t)=\omega_{0} \sum_{i} b_{i}^{\dagger} b_{i}+2 g \operatorname{Re} \phi(t) \sum_{i}\left(b_{i}+b_{i}^{\dagger}\right) .
\end{aligned}
$$

The time-dependent variables $\phi(t), X(t)$, and $n_{\alpha}(t)$ may be calculated from the Heisenberg equations of motion. We thus obtain the equations

$$
\begin{aligned}
& \frac{\partial\left\langle\boldsymbol{S}_{k}(t)\right\rangle}{\partial t}=\boldsymbol{B}_{k}(t) \times\left\langle\boldsymbol{S}_{k}(t)\right\rangle \\
& \frac{\partial\left\langle S_{k}^{0}(t)\right\rangle}{\partial t}=0 \\
& \frac{\partial X(t)}{\partial t}=\omega_{0} P(t) \\
& \frac{\partial P(t)}{\partial t}=-\omega_{0} X(t)-4 g \operatorname{Re} \phi(t),
\end{aligned}
$$

where $P(t)=i\left\langle b_{i}^{\dagger}-b_{i}\right\rangle$ is the momentum of the phonon. We solve Eqs. (14) numerically using the Runge-Kutta fourth-order method, and substitute the solutions into

$$
\left(\begin{array}{cc}
n_{0}(t) & \phi^{*}(t) \\
\phi(t) & n_{1}(t)
\end{array}\right)=\frac{1}{N} \sum_{k}\left[\left\langle\boldsymbol{S}_{k}(t)\right\rangle \cdot \boldsymbol{\sigma}+\left\langle S_{k}^{0}(t)\right\rangle \sigma_{0}\right]
$$

to obtain the excitonic order parameter and the number of electrons.

\section{Equilibrium state}

To solve the above differential equations, we need to set the initial conditions. At $t=0$, where the external field is absent, the system is in equilibrium. Since we consider the system at zero temperature, the phonons are at rest, or $P(0)=0$. From Eq. (14d), the phonons satisfy $X(0)=$ $-\frac{4 g}{\omega_{0}} \phi(0)$. We note that the excitonic order parameter is real when the electron-phonon coupling is present 12 , 36.

Diagonalizing Eq. (12), we obtain

$$
H_{\mathrm{e}}^{\mathrm{MF}}(t)=\sum_{k}\left(E_{k,+}(t) \gamma_{k,+}^{\dagger} \gamma_{k,+}+E_{k,-}(t) \gamma_{k,-}^{\dagger} \gamma_{k,-}\right)
$$

where $\gamma_{k, \pm}\left(\gamma_{k, \pm}^{\dagger}\right)$ is the annihilation (creation) operator of the quasiparticle with dispersions $E_{k, \pm}(t)=$ $\left[B_{k}^{0}(t) \pm\left|\boldsymbol{B}_{k}(t)\right|\right] / 2$. At $t=0$, the expectation value of the pseudospin is given by

$$
\begin{aligned}
& \left\langle S_{k}^{\gamma}(0)\right\rangle= \\
& \begin{cases}\frac{B_{k}^{\gamma}(0)}{2\left|\boldsymbol{B}_{k}(0)\right|}\left[f\left(E_{k,+}(0)\right)-f\left(E_{k,-}(0)\right)\right] & (\gamma=x, y, z) \\
\frac{1}{2}\left[f\left(E_{k,+}(0)\right)+f\left(E_{k,-}(0)\right)\right] & (\gamma=0)\end{cases}
\end{aligned}
$$

where $f(E)$ is the Fermi distribution function. Solving the self-consistent equations for the pseudospins, we obtain Eq. (17) with Eqs. (10) and (15) at $t=0$, which we use as the initial conditions for Eqs. (14). The calculated quasiparticle band dispersions at $t=0$ are shown in Figs. 11(b) and 1). 

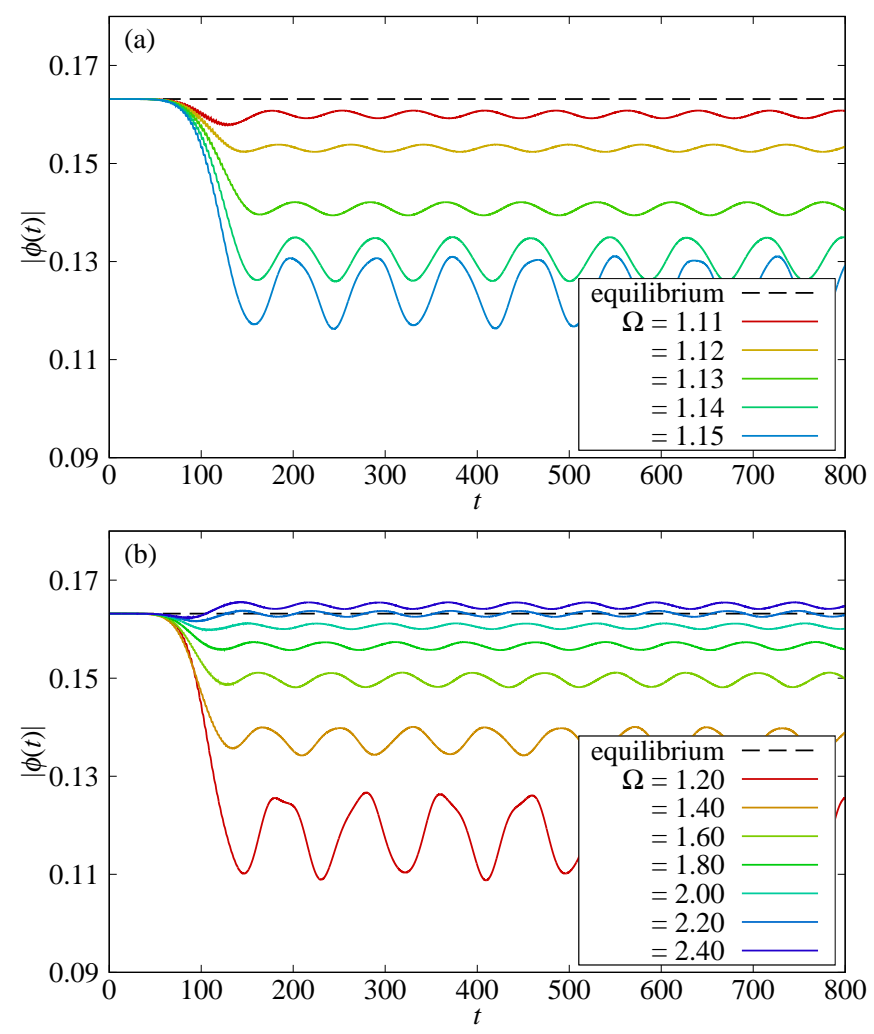

FIG. 2. Calculated time evolution of the absolute value of the excitonic order parameter $|\phi(t)|$ for a variety of frequency of the laser pulse $\Omega$ at $A_{0}=0.05$. The BEC (or semiconducting) regime is assumed. (a) The case where $\Omega$ is smaller than the quasiparticle band gap 1.15 and (b) the case where $\Omega$ is larger than the quasiparticle band gap.

\section{RESULTS OF CALCULATIONS}

Here, we discuss the results of calculations mainly when the system is semiconducting in the normal phase. Our parameter values give the quasiparticle band gap of size 1.15 in the presence of the excitonic order, which is considerably enhanced in comparison to the band gap of a size 0.88 in the absence of the excitonic order [see Fig. 1(b)]. We also discuss the results when the system is semimetallic in the last part of this section. All the calculations are made at absolute zero temperature.

\section{A. Evolution of the order parameter}

First, let us discuss the evolution of the excitonic order parameter. In Fig. 2] we show the results for the time evolution of the excitonic order parameter. We find in Fig. 2(a) that, when the frequency of the laser light is smaller than the band gap, the order parameter remains unchanged against the laser pulse. This is because the pulse cannot excite the electrons in the valence band to the conduction band. Once the frequency
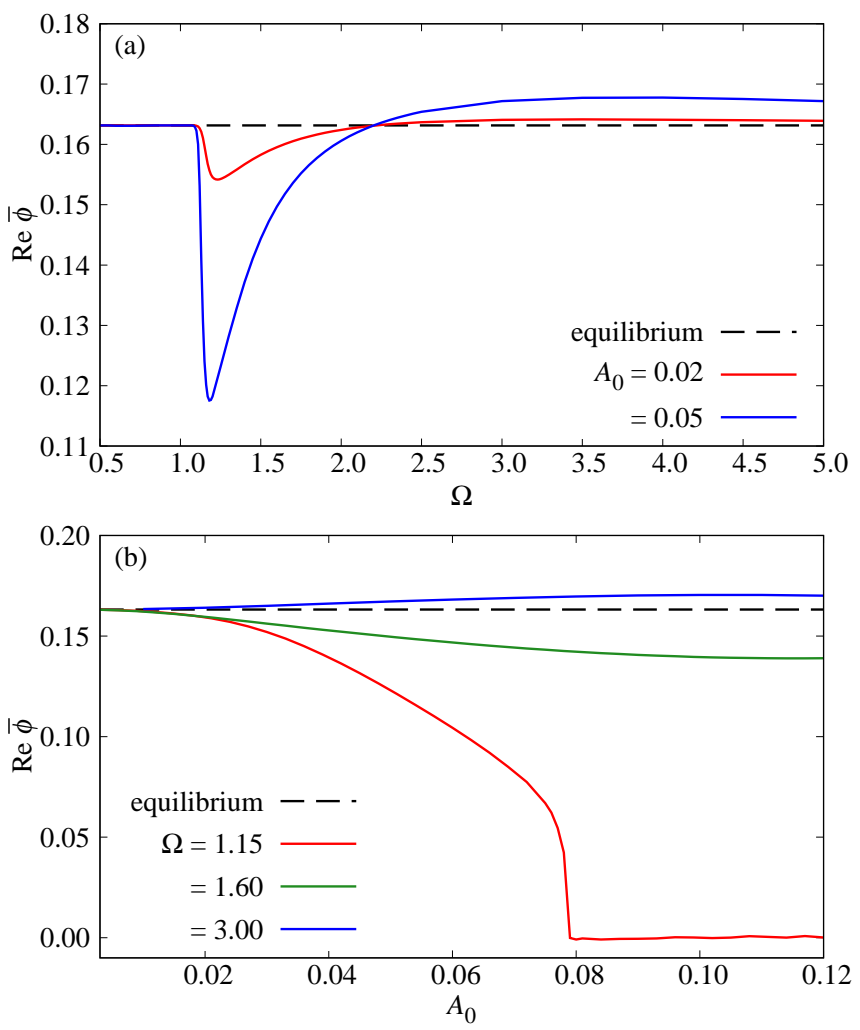

FIG. 3. Time averages of the real part of the excitonic order parameter Re $\bar{\phi}$ calculated as a function of (a) the frequency of the laser light $\Omega$ and (b) intensity of the laser light $A_{0}$.

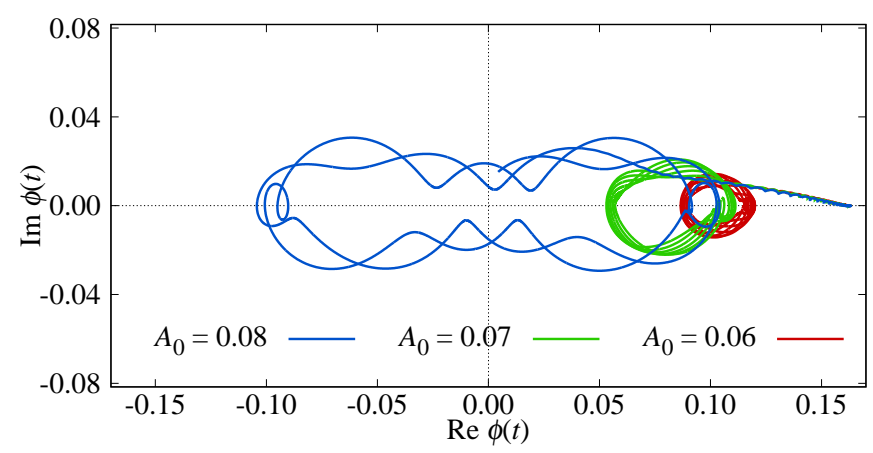

FIG. 4. Calculated trajectory of the excitonic order parameter in the complex plane for $0 \leq t<800$. The laser pulse is applied at $t=0$, where $\operatorname{Re} \phi(t)=0.163$ and $\operatorname{Im} \phi(t)=0$. We assume $\Omega=1.15$.

of the laser light becomes comparable to the size of the band gap, the excitations of electrons occur and the order parameter drastically changes as seen in Figs. 2(a) and 2 (b). After the laser pulse passes through the system, the absolute value of the order parameter decreases rapidly in comparison to the equilibrium value, and it oscillates with the frequency of the phonon. This result indicates that the excitonic order partially melts by the laser pulse. Note that, since the electron-electron cor- 
relations and electron-phonon couplings are treated in the mean-field approximation, the thermalization process of the nonequilibrium state after the pulse light passes through the system [37 40] is excluded in our calculations. As a result, the order parameter oscillates persistently without damping.

On the other hand, when the frequency of the laser light is much larger than the size of the band gap, we find that the opposite result occurs. As seen in Fig. 2(b), we find that, after the laser pulse passes through the system, the absolute value of the order parameter is enhanced. This result can be explained as the effect of the Hartree shift [25]: The strong excitations induced by the pulse lead to the reduction of the number of valence electrons and increase in the number of conduction electrons, which reduces the size of the band gap by the Hartree shift, and therefore the excitonic order parameter is enhanced.

To see the dependence of the excitonic order parameter on the frequency and intensity of the laser pulse more clearly, we introduce the time average of the order parameter $\bar{\phi}=\frac{1}{t_{2}-t_{1}} \int_{t_{1}}^{t_{2}} \phi(t) d t$, which is calculated in a sufficiently long time interval between $t_{1}$ and $t_{2}$ after the pulse is applied. The oscillations due to the phonons are thus obliterated. In Fig. 3(a), we show the real part of the averaged order parameter $\operatorname{Re} \bar{\phi}$ as a function of the frequency of the laser light. We find that, while the order parameter is suppressed for the frequencies slightly larger than the band gap, it is enhanced for the frequencies much larger than the band gap. This result can be interpreted as the competition between the melting of the excitonic order and the reduction of the band gap caused by the Hartree shift.

We also calculate the real part of the averaged order parameter as a function of the intensity of the laser light. As shown in Fig. 3(b), we find that, when the frequency of the light is around the size of the band gap, there appears a critical value of the intensity at which the averaged order parameter completely vanishes, retaining only the oscillation of the phonons. Note that, when the frequency of the laser light is much larger than the band gap, such suppression of the excitonic order does not occur.

Although not shown here, we also calculate the time average of the phonon displacement $\bar{X}$ and find that the behavior is very similar to that of the order parameter $\bar{\phi}$ shown in Fig. 3 ,

We can understand the present result clearly from the trajectory of the excitonic order parameter in the complex plane. The results are shown in Fig. 4 , where we find the following: At $t=0$, the excitonic order parameter is a real number since the electron-phonon coupling term in the Hamiltonian fixes the phase of the order parameter to zero [12, 36]. After the laser pulse is applied, the excitonic order parameter goes around a certain point where the imaginary part is zero and the real part is smaller than that in the equilibrium state. As the intensity of the laser pulse increases, the central point around which the order parameter oscillates approaches the origin, and finally it
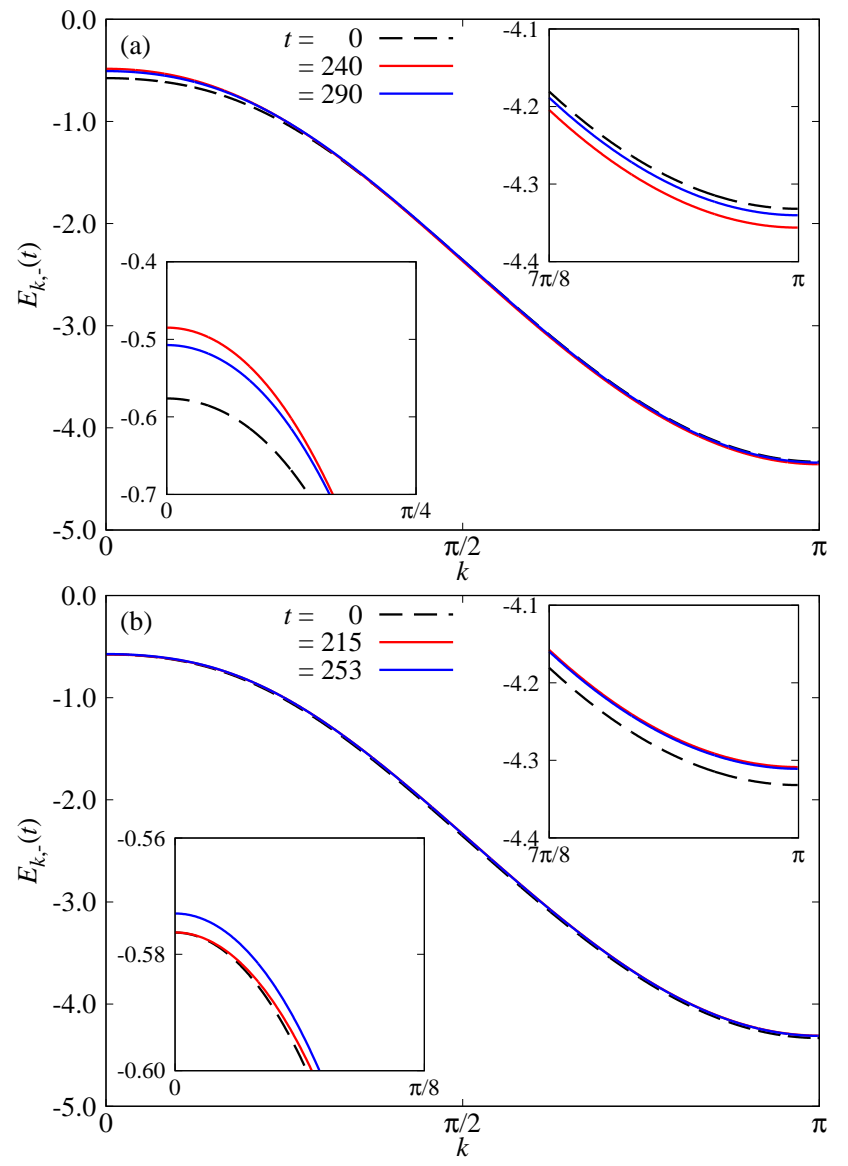

FIG. 5. Quasiparticle band dispersion below the Fermi level $E_{k,-}(t)$ calculated at time $t$. We assume (a) $A_{0}=0.05$ and $\Omega=1.15$ and (b) $A_{0}=0.05$ and $\Omega=3$. The dashed line indicates the quasiparticle band dispersion at $t=0$ (or in the equilibrium state). Insets enlarge the dispersions. At $t>0$, the dispersion oscillates between the red solid line and blue solid line.

reaches the origin when the intensity become larger than the critical value. Thus, the strong laser pulse destroys the excitonic order.

\section{B. Quasiparticle band dispersion}

We calculate the quasiparticle band dispersion in the time-dependent mean-field approximation of the model after the laser pulse is applied. The time- and angleresolved photoemission spectroscopy experiment can in principle observe this dispersion. The results are illustrated in Figs. 5(a) and 5(b). We find in Fig. 5(a) that the size of the energy gap decreases when the frequency of the laser light is slightly larger than the energy gap (or at $\Omega=1.15$ ). This is because the laser pulse leads to the partial melting of the excitonic order as shown in Fig. 3(b), of which the behavior seems consistent with recent experiment [20]. 

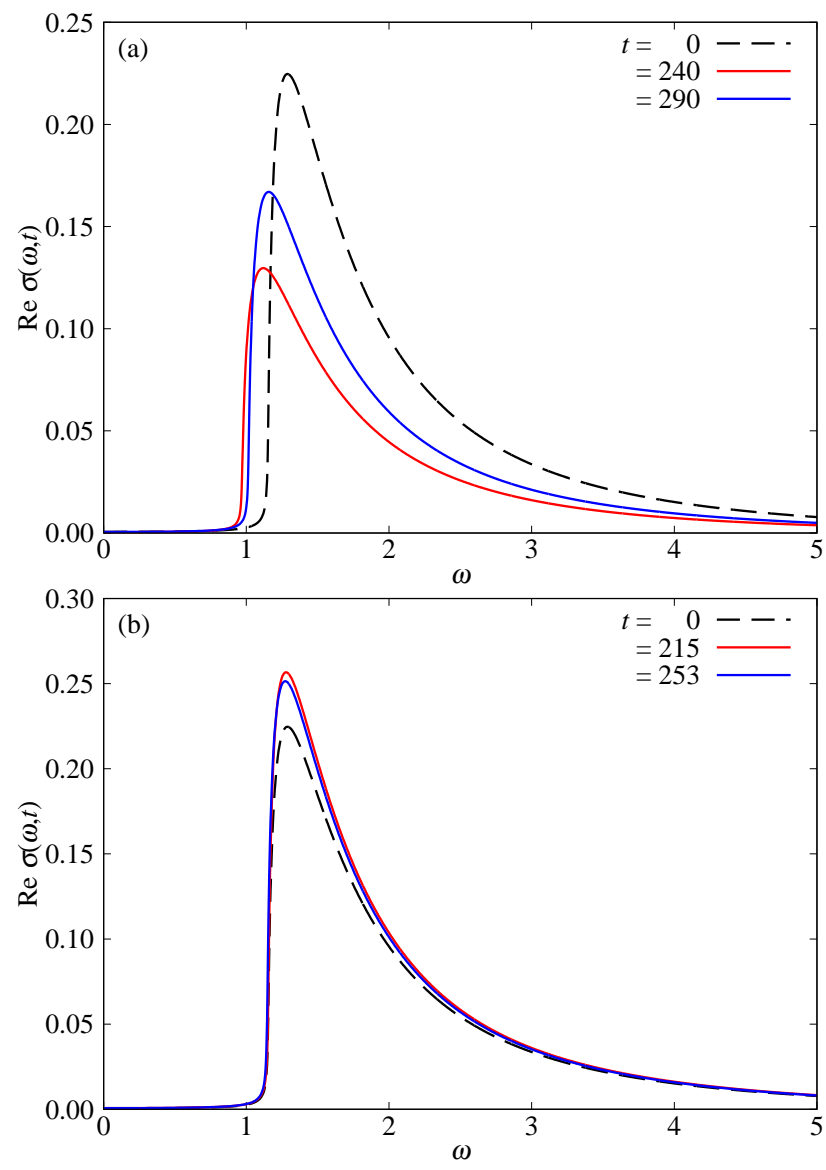

FIG. 6. Real part of the optical conductivity spectrum $\operatorname{Re} \sigma(\omega, t)$ calculated at time $t$. The dashed line indicates the optical conductivity spectrum at $t=0$ (or in the equilibrium state). We assume (a) $A_{0}=0.05$ and $\Omega=1.15$ and (b) $A_{0}=0.05$ and $\Omega=3$. At $t>0$, the spectrum oscillates between the red solid line and blue solid line.

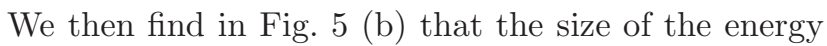
gap also decreases even when the frequency of the laser light is much larger than the energy gap (or at $\Omega=3$ ). Thus, the top of the valence band goes up in the entire frequency region of the pulse light. However, the bottom of the valence band behaves differently depending on the frequency of the laser light: it goes down at $\Omega=1.15$ but it goes up at $\Omega=3$.

\section{Optical conductivity spectrum}

To make a connection between our theory and experiment, we calculate the real part of the optical conductivity spectrum $\operatorname{Re} \sigma(\omega, t)$ measured at the frequency $\omega$ of the probe light after the pulse light passes through the system. The assumption used here is that the doubletime retarded response that is actually required in a true pump-probe setting [41 43] can be approximated by a single-time, instantaneous response for a quasi-steady state after pumping [44]. In this approximation, the real part of the optical conductivity of the present nonequilibrium state at a certain moment $t$ is given by

$$
\begin{array}{r}
\operatorname{Re} \sigma(\omega, t)=-\frac{\pi}{N \omega} \sum_{k}\left[f\left(E_{k,+}(t)\right)-f\left(E_{k,-}(t)\right)\right] \\
\times\left|J_{+-}(k, t)\right|^{2} \delta\left(E_{k,+}(t)-E_{k,-}(t)-\omega\right),
\end{array}
$$

where

$$
J_{+-}(k, t)=2 e a \frac{B_{k}^{x}(t)+i B_{k}^{y}(t)}{\left|\boldsymbol{B}_{k}(t)\right|} \sin k a
$$

is the off-diagonal matrix element of the electric current. When the excitonic order is absent, the optical conductivity completely vanishes in the present model. This is because the orbital off-diagonal elements of the current operator are all zero due to the orthogonality of the orbitals, prohibiting the excitations of electrons from the valence to the conduction bands. Only when the excitonic order appears, or the hybridization between the valence and conduction bands occurs, the optical conductivity acquires the finite spectral weight.

The calculated results are shown in Fig. 6 at $\Omega=1.15$ and $\Omega=3$. We find that the optical conductivity spectrum acquires the finite spectral weight for the probe frequency $\omega$ larger than the quasiparticle band gap. At $\Omega=1.15$, where the excitonic order is suppressed and the size of the band gap is reduced, we find that the peak position of the optical conductivity spectrum shifts to lower energy side and the peak height decreases in comparison to that of the equilibrium state [see Fig. 6(a)]. At $\Omega=3$, where the excitonic order is enhanced but the size of the band gap remains nearly unchanged, we find that the peak position of the optical conductivity spectrum remains unchanged but the peak height slightly increases in comparison to that of the equilibrium state [see Fig. 6(b)].

We note again that, in the actual measurement of the optical conductivity in the nonequilibrium state, the spectra may be affected by the phonon oscillations, so that the double-time retarded response should be taken into account in future improved calculations.

\section{Semimetallic case}

Finally, let us discuss the case where the quasiparticle band structure is semimetallic in the normal state (or in the absence of the excitonic order). To see this, we calculate the time average of the real part of the excitonic order parameter $\operatorname{Re} \bar{\phi}$ as a function of the level difference $D=\Delta_{0}-\Delta_{1}$. The semiconducting and semimetallic regions in the normal state are separated at $D=1.61$. The calculated results are summarized as a phase diagram, which is shown in Fig. 7 . We find that the excitonic order is not stable at $D>2.20$, that either the suppression or enhancement of the excitonic order occurs depending 


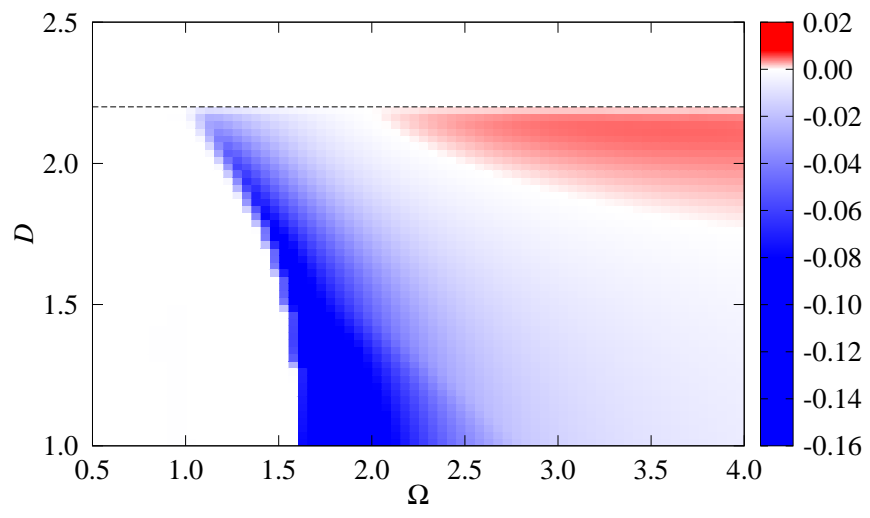

FIG. 7. Contour plot of the time average of the real part of the excitonic order parameter $\operatorname{Re} \bar{\phi}$ calculated in the parameter space of $(D, \Omega)$ at $U=2.8$. We assume $A_{0}=0.05$. The excitonic order is suppressed (enhanced) in the blue (red) region of the parameter space.

on the frequency of the laser light in the semiconducting region (or at $1.61<D<2.20$ ) as shown in Fig. 33(a), and that only the suppression of the order parameter occurs in the semimetallic region (or at $D<1.61$ ). This result is consistent with the preceding study assuming the semimetallic band structure [24].

\section{SUMMARY AND DISCUSSION}

In summary, we have studied the nonequilibrium dynamics of excitonic insulator states using the spinless two-orbital model with phonon degrees of freedom in the time-dependent mean-field approximation. Unlike preceding studies where the dipole transition of electrons between the valence and conduction bands was assumed, we have introduced the pulse light as a time-dependent vector potential via the Peierls phase in the Hamiltonian.

We have then found that, in the BEC regime where the normal state is semiconducting, the excitonic order is suppressed when the frequency of the pulse light is slightly larger than the band gap, while the order is enhanced when the frequency of the pulse is much larger than the band gap. We have moreover found that the excitonic order is completely destroyed in the former situation if the intensity of the pulse is sufficiently strong. In the BCS regime where the normal state is semimetal- lic, we have found that the excitonic order is always suppressed, irrespective of the frequency of the pulse light. We have also calculated the time-dependent quasiparticle band dispersion and optical conductivity spectrum of the model.

Finally, let us discuss possible experimental significance of our results, taking a quasi-1D direct-gap semiconductor $\mathrm{Ta}_{2} \mathrm{NiSe}_{5}$ as an example. The energy of the laser light so far used in experiment is $1.55 \mathrm{eV}$ [20 23], which is very large in comparison with the observed band gap $160 \mathrm{meV}$ [10, 45, 46]. Thus, our two-band model seems to be too simple to take into account the excitations in such a high-energy scale where other orbitals such as Se $4 p$ become relevant. In other words, the use of lower-energy laser lights may be more informative to clarify the nonequilibrium dynamics of $\mathrm{Ta}_{2} \mathrm{NiSe}_{5}$ in the lowest energy scales. Also, it was pointed out that $\mathrm{Ta}_{2} \mathrm{NiSe}_{5}$ is in the strong coupling BEC regime, despite the fact that the noninteracting band structure is semimetallic [47. Such a situation cannot be treated in our simple mean-field approximation. Nevertheless, we may point out that our calculations could yield some experimental aspects such as the enhancement of the excitonic order [20, 21], coherent order parameter oscillations [22, 23], as well as the insulator-to-metal transitions reported recently [48]. The improved method of calculations beyond the mean-field approximation based on more realistic models will be required for future quantitative studies of the nonequilibrium dynamics of the excitonic insulator states. We may note here that, in the case of superconductivity under periodically oscillating phonons driven by an external field, the order parameter is reduced in the dynamical mean-field-theory calculation [4], while it is enhanced in the effective electron-electron interaction scheme [50, 51]; the opposite results are obtained depending on the approximations used. Thus, a careful treatment of strongly interacting systems will be required in future calculations of nonequilibrium phenomena observed in the excitonic phases as well.

\section{ACKNOWLEDGMENTS}

We thank T. Kaneko, T. Mizokawa, Y. Murakami, K. Okazaki, Y. Yamada, and K. Yonemitsu for enlightening discussions. This work was supported in part by a Grant-in-Aid for Scientific Research (No. JP17K05530) from JSPS of Japan.
[1] D. Fausti, R. I. Tobey, N. Dean, S. Kaiser, A. Dienst, M. C. Hoffmann, S. Pyon, T. Takayama, H. Takagi, and A. Cavalleri, Science 331, 189 (2011).

[2] M. Mitrano, A. Cantaluppi, D. Nicoletti, S. Kaiser, A. Perucchi, S. Lupi, P. Di Pietro, D. Pontiroli, M. Riccò, S. R. Clark, D. Jaksch, and A. Cavalleri, Nature 530, 461 (2016).
[3] L. Perfetti, P. A. Loukakos, M. Lisowski, U. Bovensiepen, H. Berger, S. Biermann, P. S. Cornaglia, A. Georges, and M. Wolf, Phys. Rev. Lett. 97, 067402 (2006).

[4] F. Schmitt, P. S. Kirchmann, U. Bovensiepen, R. G. Moore, L. Rettig, M. Krenz, J.-H. Chu, N. Ru, L. Perfetti, D. H. Lu, M. Wolf, I. R. Fisher, and Z.-X. Shen, Science 3211649 (2008). 
[5] D. Jérome, T. M. Rice, and W. Kohn, Phys. Rev. 158, 462 (1967).

[6] B. I. Halperin and T. M. Rice, Rev. Mod. Phys. 40, 755 (1968).

[7] H. Cercellier, C. Monney, F. Clerc, C. Battaglia, L. Despont, M. G. Garnier, H. Beck, P. Aebi, L. Patthey, H. Berger, and L. Forró, Phys. Rev. Lett. 99, 146403 (2007).

[8] A. Kogar, M. S. Rak, S. Vig, A. A. Husain, F. Flicker, Y. Il Joe, L. Venema, J. Macdougall, T. C. Chiang, E. Fradkin, J. Van Wezel, P. Abbamonte, and S. Materials, Science 358, 1314 (2017).

[9] Y. Wakisaka, T. Sudayama, K. Takubo, T. Mizokawa, M. Arita, H. Namatame, M. Taniguchi, N. Katayama, M. Nohara, and H. Takagi, Phys. Rev. Lett. 103, 026402 (2009).

[10] K. Seki, Y. Wakisaka, T. Kaneko, T. Toriyama, T. Konishi, T. Sudayama, N. L. Saini, M. Arita, H. Namatame, M. Taniguchi, N. Katayama, M. Nohara, H. Takagi, T. Mizokawa, and Y. Ohta, Phys. Rev. B 90, 155116 (2014).

[11] V. N. Phan, K. W. Becker, and H. Fehske, Phys. Rev. B 88, 205123 (2013).

[12] B. Zenker, H. Fehske, and H. Beck, Phys. Rev. B 90, 195118 (2014).

[13] T. Kaneko, T. Toriyama, T. Konishi, and Y. Ohta, Phys. Rev. B 87, 035121 (2013); Phys. Rev. B 87, 199902(E) (2013).

[14] K. Sugimoto, T. Kaneko, and Y. Ohta, Phys. Rev. B 93, 041105 (2016).

[15] T. Kaneko, Y. Ohta, and S. Yunoki, Phys. Rev. B 97, 155131 (2018).

[16] T. Rohwer, S. Hellmann, M. Wiesenmayer, C. Sohrt, A. Stange, B. Slomski, A. Carr, Y. Liu, L. M. Avila, M. Kalläne, S. Mathias, L. Kipp, K. Rossnagel, and M. 1 Bauer, Nature 471, 490 (2011).

[17] E. Möhr-Vorobeva, S. L. Johnson, P. Beaud, U. Staub, R. De Souza, C. Milne, G. Ingold, J. Demsar, H. Schaefer, and A. Titov, Phys. Rev. Lett. 107, 036403 (2011).

[18] S. Hellmann, T. Rohwer, M. Kallüe, K. Hanff, C. Sohrt, A. Stange, A. Carr, M. M. Murnane, H. C. Kapteyn, L. Kipp, M. Bauer, and K. Rossnagel, Nature Commun. 3, 1069 (2012).

[19] C. Monney, M. Puppin, C. W. Nicholson, M. Hoesch, R. T. Chapman, E. Springate, H. Berger, A. Magrez, C. Cacho, R. Ernstorfer, and M. Wolf, Phys. Rev. B 94, 165165 (2016).

[20] S. Mor, M. Herzog, D. Golež, P. Werner, M. Eckstein, N. Katayama, M. Nohara, H. Takagi, T. Mizokawa, C. Monney, and J. Stähler, Phys. Rev. Lett. 119, 086401 (2017).

[21] S. Mor, M. Herzog, J. Noack, N. Katayama, M. Nohara, H. Takagi, A. Trunschke, T. Mizokawa, C. Monney, and J. Stähler, Phys. Rev. B 97, 115154 (2018).

[22] D. Werdehausen, T. Takayama, G. Albrecht, Y. Lu, H. Takagi, and S. Kaiser, J. Phys.: Condens. Matter 30, 305602 (2018).

[23] D. Werdehausen, T. Takayama, M. Höppner, G. Albrecht, A. W. Rost, Y. Lu, D. Manske, H. Takagi, and S. Kaiser, Sci. Adv. 4, eaap8652 (2018).

[24] D. Golež, P. Werner, and M. Eckstein, Phys. Rev. B 94, 035121 (2016).

[25] Y. Murakami, D. Golež, M. Eckstein, and P. Werner, Phys. Rev. Lett. 119, 247601 (2017).
[26] Y. Tanaka, M. Daira, and K. Yonemitsu, Phys. Rev. B 97, 115105 (2018).

[27] P. Wissgott, J. Kuneš, A. Toschi, and K. Held, Phys. Rev. B 85, 205133 (2012).

[28] D. Ihle, M. Pfafferott, E. Burovski, F. X. Bronold, and H. Fehske, Phys. Rev. B 78193103 (2008).

[29] B. Zenker, D. Ihle, F. X. Bronold, and H. Fehske, Phys. Rev. B 83, 235123 (2011).

[30] K. Seki, R. Eder, and Y. Ohta, Phys. Rev. B 84, 245106 (2011).

[31] S. Ejima, T. Kaneko, Y. Ohta, and H. Fehske, Phys. Rev. Lett. 112, 026401 (2014).

[32] J. K. Freericks, O. P. Matveev, W. Shen, A. M. Shvaika, and T. P. Devereaux, Phys. Scr. 92, 034007 (2017).

[33] H. Matsueda, S. Sota, T. Tohyama, and S. Maekawa, J. Phys. Soc. Jpn. 81, 013701 (2012).

[34] R. A. Barankov, L. S. Levitov, and B. Z. Spivak, Phys. Rev. Lett. 93, 160401 (2004).

[35] E. A. Yuzbashyan, B. L. Altshuler, V. B. Kuznetsov, and V. Z. Enolskii, Phys. Rev. B 72, 220503(R) (2005).

[36] T. Kaneko, B. Zenker, H. Fehske, and Y. Ohta, Phys. Rev. B 92, 115106 (2015).

[37] N. Tsuji, M. Eckstein, and P. Werner, Phys. Rev. Lett. 110, 136404 (2013).

[38] A. F. Kemper, M. A. Sentef, B. Moritz, J. K. Freericks, and T. P. Devereaux, Phys. Rev. B 92, 224517 (2015).

[39] M. Schüler, M. Eckstein, and P. Werner, Phys. Rev. B 97, 245129 (2018).

[40] M. A. Sentef, A. F. Kemper, A. Georges, and C. Kollath, Phys. Rev. B 93, 144506 (2016).

[41] M. Eckstein and M. Kollar, Phys. Rev. B 78, 205119 (2008).

[42] Z. Lenarčič, D. Golež, J. Bonča, and P. Prelovšek, Phys. Rev. B 89, 125123 (2014).

[43] C. Shao, T. Tohyama, H.-G. Luo, and H. Lu, Phys. Rev. B 93, 195144 (2016).

[44] R. Fukaya, Y. Okimoto, M. Kunitomo, K. Onda, T. Ishikawa, S. Koshihara, H. Hashimoto, S. Ishihara, A. Isayama, H. Yui, and T. Sasagawa, Nature Commun. 6, 8519 (2015).

[45] Y. F. Lu, H. Kono, T. I. Larkin, A. W. Rost, T. Takayama, A. V. Boris, B. Keimer, and H. Takagi, Nature Commun. 8, 14408 (2017).

[46] T. I. Larkin, A. N. Yaresko, D. Pröpper, K. A. Kikoin, Y. F. Lu, T. Takayama, Y.-L. Mathis, A. W. Rost, H. Takagi, B. Keimer, and A. V. Boris, Phys. Rev. B 95, 195144 (2017).

[47] K. Sugimoto, S. Nishimoto, T. Kaneko, and Y. Ohta, Phys. Rev. Lett. 120, 247602 (2018).

[48] K. Okazaki, Y. Ogawa, T. Suzuki, T. Yamamoto, T. Someya, S. Michimae, M. Watanabe, Y. Lu, M. Nohara, H. Takagi, N. Katayama, H. Sawa, M. Fujisawa, T. Kanai, N. Ishii, J. Itatani, T. Mizokawa, and S. Shin, Nature Commun. 9, 4322 (2018).

[49] Y. Murakami, N. Tsuji, M. Eckstein, and P. Werner, Phys. Rev. B 96, 045125 (2017).

[50] M. Knap, M. Babadi, G. Refael, I. Martin, and E. Demler, Phys. Rev. B 94, 214504 (2016).

[51] A. Komnik and M. Thorwart, Eur. Phys. J. B 89, 244 (2016). 